\title{
Muscle activity during the incline shoulder press in relation to the exercise intensity
}

\author{
Katarzyna Stronska ${ }^{1}$, Paulina Bojacz ${ }^{1}$, Artur Golas' ${ }^{1}$, Adam Maszczyk², Adam Zajac ${ }^{1}$, \\ and Petr Stastny ${ }^{3, *}$ \\ ${ }^{1}$ Department of Sports Training, The Jerzy Kukuczka Academy of Physical Education in Katowice, Katowice, Poland; \\ ${ }^{2}$ Department of Methodology and Statistic, The Jerzy Kukuczka Academy of Physical Education in Katowice, Katowice, \\ Poland; and ${ }^{3}$ Faculty of Physical Education and Sport, Charles University, Prague, Czech Republic
}

Copyright: (c) 2018 K. Stronska et al. This is an open access article licensed under the Creative Commons Attribution License (http://creativecommons.org/licenses/by/4.0/).

\begin{abstract}
Background: The incline machine shoulder press (IMSP) is an alternative resistance exercise to the bench press associated with throwing performance. The muscle activity during IMSP has not yet been described in females and at different exercises intensities. Objectives: The aim of this present study was to investigate changes in the activity of prime movers during the IMSP in relation to the exercise intensity in female athletes. Methods: Eight female athletes experienced in resistance training were screened for peak electromyography amplitude normalized for maximum voluntary isometric contraction during the IMSP with increasing loads of $40 \%, 60 \%, 80 \%$ and $100 \%$ of 1 repetition maximum (1RM). The selected muscles were the anterior deltoid (AD), pectoralis major (PM), triceps brachii long head (TBlong) and triceps brachii lateral head (TBlat). Results: The results of Friedman ANOVA showed increased muscle activity along with exercise intensity in all prime movers $(p<.001)$. The Kruskal-Wallis ANOVA showed inter-muscle differences at exercise intensity of 40\% 1RM $\left(p=.004, \eta_{\mathrm{p}}^{2}=.52\right), 60 \% 1 \mathrm{RM}\left(p=.005, \eta_{\mathrm{p}}^{2}=.55\right), 80 \%$ $1 \mathrm{RM}\left(p=.035, \eta_{\mathrm{p}}^{2}=.46\right)$ and $100 \% 1 \mathrm{RM}\left(p=.002, \eta_{\mathrm{p}}^{2}=.52\right)$, where TBlat showed lower activity than other muscle groups at each exercise intensity. The TBlong showed lower activity than the PM and AD at 40\% 1 RM, and the PM activity was lower than that of $\mathrm{AD}$ and TBlong at 100\% 1RM. Conclusions: The IMSP is an exercise which activated $\mathrm{AD}$ and TBlong more than PM and TBlat during maximal lifting effort. Therefore, the IMSP should be understood as the exercise which might substantially overload the AD and TBlong during maximal lifts. Coaches should use the IMSP if they desire to activate TBlong more than TBlat during upper limb pressing.
\end{abstract}

Keywords: resistance exercise, strength, muscle action, upper body, exercise intensity, electromyography

\section{Introduction}

In modern sports, one of the main elements of the training process is the analysis of fundamental movement patterns as a basis for development of the athlete's strength abilities. Depending on anthropometric variables, gender and movement technique, the muscular activity patterns vary during the upper limb pressing exercises, yet most research confirms that three muscle groups are involved as prime movers in upper limb pressing exercises: pectoralis major (PM), anterior deltoid (AD) and triceps brachii (TB) (Gołaś, Maszczyk, Pietraszewski, et al., 2018; Gołaś et al., 2017; Król \& Gołaś, 2017; Stastny et al., 2017).

\footnotetext{
* Address for correspondence: Petr Stastny, Department of Sport Games, Faculty of Physical Education and Sport, Charles University, José Martího 269/31, 16252 Prague 6, Czech Republic. E-mail: stastny@ftvs.cuni.cz
}

Knowledge of muscle activity during the upper limb pressing movements can help determine which exercise variations are effective for increasing an athlete's performance or neuromuscular adaptation, and which variation can strengthen or overload specific muscles during training, therapy or reconditioning.

Besides the bench press (BP), numerous variations of pressing exercises with different muscle activity are used in individual sports, team sports and general conditioning. The standing cable press has been found to activate the $\mathrm{AD}$ and $\mathrm{PM}$, but performance seems limited by the activation and neuromuscular coordination of torso muscles (Santana, Vera-Garcia, \& McGill, 2007). The dumbbell chest press exercise has been found to have lower TB activity when compared with the BP (van den Tillaar \& Sæterbakken, 2012) and free weight BP has been found to have greater activation of medial deltoid in comparison to the Smith 
machine BP (McCaw \& Friday, 1994; Schick et al., 2010). Moreover, the activity of the clavicular part of $\mathrm{PM}$ and $\mathrm{AD}$ increases along with bench inclination (Barnett, Kippers, \& Turner, 1995; Lauver, Cayot, \& Scheuermann, 2016; Trebs, Brandenburg, \& Pitney, 2010) up to the transition to shoulder press. Although studies on BP variations are well described in terms of exercise intensity, speed of movement, training method (Stastny et al., 2017) and grip variations (Barnett et al., 1995; Lehman, 2005), the studies on the shoulder press, including the incline machine shoulder press (IMSP) are scarce.

The incline shoulder press performed with a barbell or a machine is an alternative resistance exercise to the BP (Lantz \& McCrain, 2005), where the incline BP has been associated with throwing performance (Terzis, Karampatsos, \& Georgiadis, 2007). Therefore, there is a practical question of specific muscle contribution during IMSP. The shoulder press has been compared to different variations of the $\mathrm{BP}$, but previous research included shoulder press only at submaximal intensities $(70-80 \%$ of 1 repetition maximum) (Barnett et al., 1995; Trebs et al., 2010). Other findings showed that $\mathrm{AD}$ activity in the $\mathrm{BP}$ differs between male and female athletes (Gołaś, Maszczyk, Pietraszewski, et al., 2018), and that exercise intensity is the main factor influencing muscle activity which interact with other loading variables including exercise selection (Stastny et al., 2017).

Because the muscle activity during IMSP has not been described in females and at different exercises intensities, the aim of the present study was to investigate changes in the prime movers activity during the IMSP in relation to the exercise intensity in female athletes. We hypothesized that all prime movers will increase their activity along with exercise intensity and that $\mathrm{AD}$ would be the most active muscle at each exercise intensity.

\section{Methods}

\section{Participants}

Eight female athletes recruited from varsity teams representing different overhead disciplines (basketball, handball, shot put, and javelin), with at least one year of experience in resistance training took part in the study. During this period the participants performed IMSP exercises routinely twice a week. Their age, body mass and body height were $24.7 \pm 2.6$ years, $56.3 \pm 5.2 \mathrm{~kg}$ and $168.3 \pm 4.2 \mathrm{~cm}$, respectively. Their average $1 \mathrm{RM}$ equaled $52.5 \pm 5.5 \mathrm{~kg}$. The participants did not perform any additional resistance exercises for 72 hours prior to testing to avoid fatigue. Prior to the testing, all participants were informed verbally and in writing about the procedures, possible risks and benefits of the tests, and they provided written consent before the commencement of the study. The study received the approval of the Bioethics Committee at the Jerzy Kukuczka Academy of Physical Education in Katowice, Poland (NRSA 404054).

\section{Instrumentation}

The IMSP was performed on a Hoist shoulder press machine (Hoist Fitness Systems, Poway, CA, USA) with neutral grip position, bench angle set up on $45^{\circ}$, and adjustable footplate set up to $90^{\circ}$ of knee flexion and hip flexion. A metronome (Witnner 834, Wittner, Isny, Germany) has been used to control the pace of movement and the rest between repetitions.

An eight-channel Noraxon TeleMyo 2400 system (Noraxon USA Inc., Scottsdale, AZ, USA; 1500 Hz) was used for recording and analysis of biopotentials from the muscles (PM, AD, TB lateral and long head) using the gel-coated self-adhesive electrodes (Dri-Stick Silver circular sEMG Electrodes AE-131, NeuroDyne Medical, Cambridge, MA, USA). The adhesive couples of electrodes (11 mm contact diameter and a 2 $\mathrm{cm}$ fixed electrodes distance) were placed along the presumed direction of the underlying muscle fibers according to the recommendations by SENIAM (Hermens et al., 1999). Video recording was used for identification of the beginning and completion of the movement.

\section{Procedures}

The procedures included protocol familiarization (within one week prior to the measurement), premeasurement 1 repetition maximum (1RM) estimation (one week prior to the measurement) and the main measurement session. The pre-measurement determination of 1RM was performed according to the protocol proposed by Baechle (Baechle \& Earle, 2008), using a warm-up and progressively increasing load.

Before the experimental session, a standardized warm-up protocol was used, where athletes performed a general warm-up (5 min), using a hand cycle ergometer (heart rate of approximately $130 \mathrm{bpm}$ ), as well as 5 min of dynamic stretching. The specific part of the warm-up consisted of three sets of incline shoulder presses with the load adjusted accordingly to perform 15,10 and 5 repetitions with $3-5$ min rest interval. After the warm-up, and 10 minutes before the start of the experimental protocol, maximum voluntary isometric contraction (MVIC) of particular prime movers were performed in order to normalize electromyographic records according to the SENIAM procedure 
(Hermens et al., 1999). The TB MVIC was obtained during lying triceps extension with $90^{\circ}$ elbow flexion, the AD MVIC at $90^{\circ}$ seated arm flexion, and the PM MVIC during an isometric BP at $90^{\circ}$ elbow flexion. All MVIC tests were performed against a fixed multipress bar where the joint positions for muscle test were in accordance with the SENIAM recommendation (Hermens et al., 1999).

The main protocol included the IMSP performed in a stretch shortening cycle (SSC) in both the eccentric and concentric phases of the movement with progressively increasing load $(40,60,80$, and $100 \%$ of $1 \mathrm{RM})$ and with rest interval 3-5 min. A total of 5 repetitions were performed for each submaximal loading condition (40, 60 and 80 1RM) and three successful attempts for $1 \mathrm{RM}$. The athletes performed the eccentric part of the movement at a pace of $2 \mathrm{~s}$, and then the concentric phase (lasted for $2 \mathrm{~s}$ in submaximal and $\max 4 \mathrm{~s}$ for $1 \mathrm{RM}$ lifts) up to full extension in the elbow joints and had a $1 \mathrm{~s}$ hold in the initial position before the next repetition. Muscle activity was measured during the lowering and lifting phase of the incline shoulder press. During each repetition, the athletes were motivated verbally.

\section{Data acquisition}

The EMG activity was recorded for four muscles: PM, AD, TB lateral head (TBlat) and TB long head (TBlong) on the dominant side of the body (determined by throwing and writing hand preference). Before placing the gel-coated self-adhesive electrodes on the athlete, the skin was shaved, abraded and cleaned with alcohol. The PM electrodes were placed on sternocostal fibers, $4 \mathrm{~cm}$ medial to the axillary fold, AD electrodes were placed $1.5 \mathrm{~cm}$ distal and anterior to the acromion, and TBlong electrode was placed over the proximal part of the muscle belly, TBlat electrode was placed on the lateral part of the muscle belly, and ground electrode was placed on the olecranon. The MVIC was performed three times for 2-3 s, where best recorded value has been used for signal normalization.

The EMG analogue signals sampled at a rate of $1000 \mathrm{~Hz}$ were band pass filtered with a cut off frequency of $8 \mathrm{~Hz}$ and $450 \mathrm{~Hz}$ and smoothed using the root mean square with a sliding window function at a time constant of $25 \mathrm{~ms}$. The signal obtained during IMSP was normalized to the EMG during MVIC to be expressed as a percentage of MVIC (\%MVIC), where the peak amplitude from SSC has been used for statistical analyses.

\section{Statistical analysis}

The data was processed using ORIGINE software (Version 2018b SR0; OriginLab, Wellesley Hills, MA, USA) where statistical significance was set up at $\alpha=.05$. The intraclass correlation coefficient across 5 repetitions in submaximal loads and 3 repetitions during maximal loads for each individual was calculated to determine intra-session reliability. Friedman analyses of variance (ANOVA) were used to compare EMG between exercise intensity $(40 \%, 60 \%, 80 \%$, and $100 \% 1 \mathrm{RM})$ for each muscle group (AD, PM, TBlat, and TBlong) followed by Dunn's post hoc tests. Kruskal-Wallis ANOVA was used to compare EMG (dependent variable) between muscle groups (independent variable) at each exercise intensity followed by Dunn's post hoc tests. Only results with statistical power (type II error $1-\beta$ calculated from $n$ per group, $\mathrm{n}$ of groups and root mean square standardized effect) above .8 were accepted (Cohen, 1988). The effect size (partial eta squared $-\eta_{\mathrm{p}}^{2}$ ) of each test was calculated for all analyses and was classified according to Larson-Hall (Larson-Hall, 2010), where $\eta_{p}^{2}$ values .02, .13 , and .26 were considered as small, moderate, and large effects respectively.

\section{Results}

The within-subject reliability across repetitions resulted in intraclass correlation coefficient values ranging from .81 to .92 for all muscles and intensities, which indicate a high or very high reliability of measurement (Chandler \& Brown, 2008). Peak muscle activity of the four considered muscles during the incline shoulder press with loads of $40 \%, 60 \%, 80 \%$, and 100\% 1RM are presented as medians in Table 1 and as means in Figure 1.

The Friedmann ANOVA (Figure 1) showed differences between exercise intensities in $\mathrm{AD}\left(\chi^{2}=24\right.$, $\left.p<.001, \eta_{\mathrm{p}}^{2}=.88\right), \mathrm{PM}\left(\chi^{2}=24, p<.001, \eta_{\mathrm{p}}^{2}=.87\right)$, TBlong $\left(\chi^{2}=23, p<.001, \eta_{\mathrm{p}}^{2}=.86\right)$, and TBlat $\left(\chi^{2}=21, p<.001, \eta_{\mathrm{p}}^{2}=.80\right)$. The post hoc test showed differences between all exercise intensities in AD, PM and TBlong. In TBlat post hoc test showed differences between the $40 \%$ of $1 \mathrm{RM}$ compared to $80 \% 1 \mathrm{RM}$, $40 \%$ of $1 \mathrm{RM}$ compared to $100 \% 1 \mathrm{RM}, 60 \%$ of $1 \mathrm{RM}$ compared to $80 \%$ of $1 \mathrm{RM}$ and between $80 \%$ of $1 \mathrm{RM}$ compared to $100 \% 1 \mathrm{RM}$.

The Kruskal-Wallis ANOVA (Table 1) showed inter-muscle differences at exercise intensity of $40 \% 1 \mathrm{RM}\left(\chi^{2}=13, p=.004, \eta_{\mathrm{p}}^{2}=.52\right), 60 \% 1 \mathrm{RM}$ $\left(\chi^{2}=13, p=.005, \eta_{\mathrm{p}}^{2}=.55\right), 80 \% 1 \mathrm{RM}\left(\chi^{2}=9\right.$, $\left.p=.035, \eta_{\mathrm{p}}^{2}=.46\right)$ and $100 \% 1 \mathrm{RM}\left(\chi^{2}=15, p=.002\right.$, $\left.\eta_{\mathrm{p}}^{2}=.52\right)$, where TBlat showed lower activity than other muscle groups at each exercise intensity, TBlong showed lower activity than PM and AD during 40\% 1RM, and PM showed lower activity than $A D$ and TBlong during 1RM. 


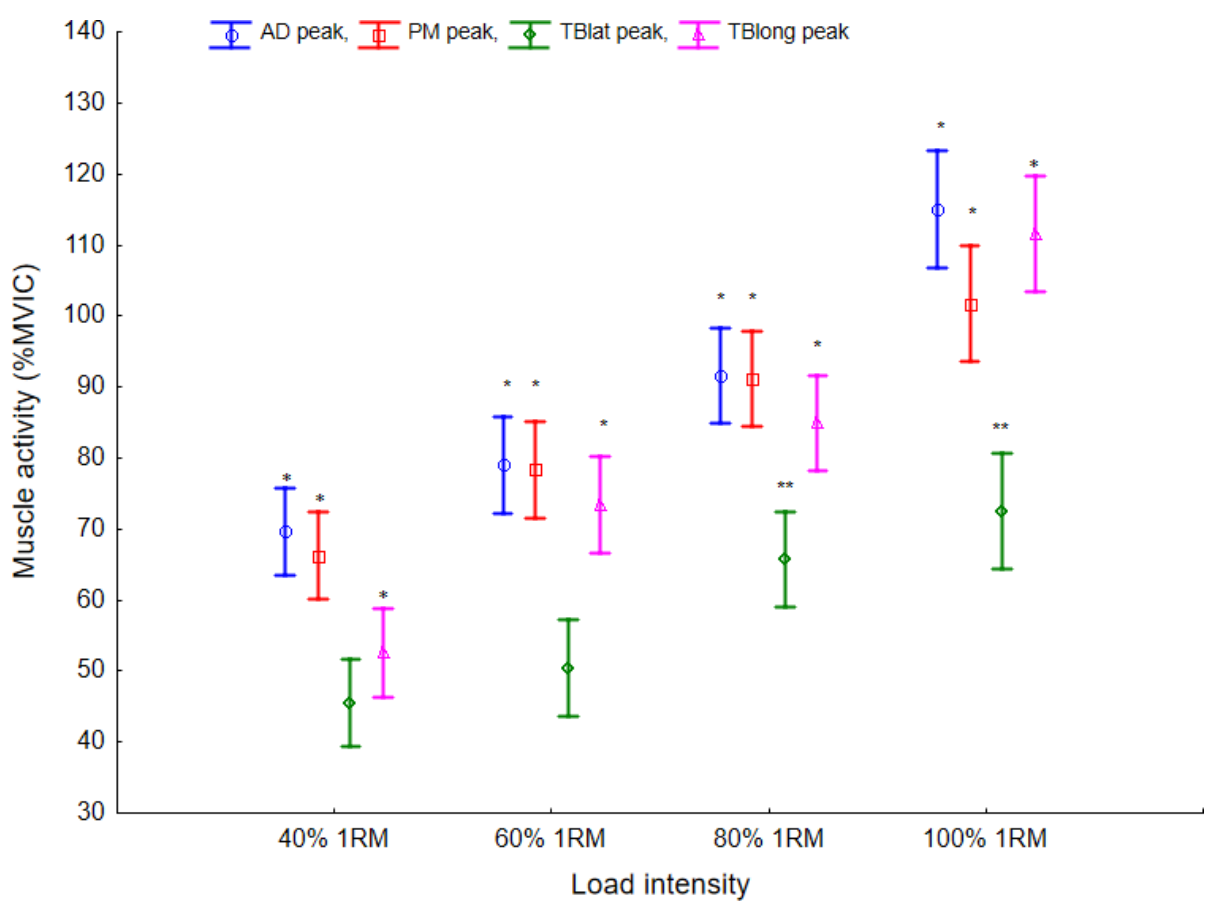

Figure 1. Differences in peak EMG amplitude activity between different exercise intensities of $40-100 \%$ of 1 repetition maximum (1RM). The values in the graph are expressed as mean and standard deviation. \%MVIC $=$ maximal voluntary isometric contraction; $\mathrm{AD}=$ anterior deltoid; $\mathrm{PM}=$ pectoralis major; $\mathrm{TBlat}=$ triceps brachii lateral head; TBlong $=$ triceps brachii long head. * indicates a significant difference by a post hoc test to other exercise intensity for the same muscle group, $* *$ indicates a significant difference by a post hoc test to $40-60 \% 1 \mathrm{RM}$ load.

Table 1

Muscle activity at various exercise intensities as a peak amplitude normalized to maximum voluntary isometric contraction

\begin{tabular}{lcccc}
\hline Muscle & $40 \% 1 \mathrm{RM}$ & $60 \% 1 \mathrm{RM}$ & $80 \% 1 \mathrm{RM}$ & $100 \% 1 \mathrm{RM}$ \\
\hline $\mathrm{AD}$ & $72(65-76)$ & $80(72-89)$ & $92(84-100)$ & $117(107-125)$ \\
$\mathrm{PM}$ & $67(57-73)$ & $79(67-89)$ & $90(81-100)$ & $103(88-109) \uparrow$ \\
TBlat & $46(43-49) \dagger$ & $50(49-53) \dagger$ & $66(60-70) \dagger$ & $72(71-76) \dagger$ \\
TBlong & $56(43-60) \ddagger$ & $73(67-80)$ & $86(81-89)$ & $112(109-118)$ \\
\hline
\end{tabular}

Note. The values are presented as median (interquartile range $25-75 \%$ ). $1 \mathrm{RM}=1$ repetition maximum; $\mathrm{AD}=$ anterior deltoid; $\mathrm{PM}=$ pectoralis major; TBlat = triceps brachii lateral head; TBlong = triceps brachii long head. $\dagger$ indicates a significant difference to all other muscle groups at the same intensity, $¥$ indicates a significant difference to AD and PM at the same intensity, $\uparrow$ indicates a significant difference to AD and TBlong at the same intensity.

\section{Discussion}

The main finding of this study is that TBlat had the smallest activity among the prime movers during IMSP at all exercise intensities. Moreover, the TBlat activity did not increase between the intensities of $40-60 \%$ $1 \mathrm{RM}$ and $80-100 \% 1 \mathrm{RM}$. Therefore, we can conclude that our hypothesis cannot be confirmed for TBlat activity. The $\mathrm{AD}$, as well as the TBlong were the most active muscle groups at maximal intensity $100 \%$ of $1 \mathrm{RM}$ in female athletes, which was hypothesized only for AD. Since the high activity measured by \%MVIC is related to motor units recruitment during resistance training (Vigotsky, Halperin, Lehman, Trajano, \& Vieira, 2017) we can expect that IMSP can elicit the prior exhaustion of $\mathrm{AD}$ and TBlong. Increased muscular activation as a result of training is most often ascribed to a combination of greater recruitment (the number of fibers involved in a muscle action), and higher rate coding of the frequency at which the motor units are stimulated (Enoka \& Duchateau, 2015). The greatest increase in activity was visible for the TBlong and the 
smallest one was observed for the TBlat This may be explained by elbow and shoulder position during the incline shoulder press, where initial arm position in this movement can alternate TB heads force contribution (Kholinne et al., 2018).

In comparison to previous studies, the differences between the IMSP and flat BP at 1RM were observed primarily in the activity of the TBlong, which was greater in IMSP than in the BP (Gołaś, Maszczyk, Pietraszewski, et al., 2018). On the other hand, the IMSP and flat BP seems to elicit similar TBlat, AD and PM activity during submaximal lifts (60-85\% 1RM; Gołaś, Maszczyk, Pietraszewski, et al., 2018), which should be considered in exercise selection to purposely overlap or avoid overlapping of exposed muscles during the training session. A lot of practitioners believe that $\mathrm{PM}$ is the key muscle group to exercise when increasing maximum strength during upper limb pressing (Barnett et al., 1995; Golas, Masczyk, Stastny, et al., 2018a). Our study, and some previous findings show that the PM increases its activity along with the lifted load during pressing, however performing $100 \%$ of $1 \mathrm{RM}$ does not elicit a higher peak in PM than other prime movers during pressing (Gołaś, Maszczyk, Pietraszewski, et al., 2018; Król \& Gołaś, 2017; Maszczyk et al., 2016). One of the explanations may be that the PM is an important prime mover for the upper limb pressing exercises responsible for overcoming the sticking region in submaximal intensity such as 6RM BP (van den Tillaar, Saeterbakken, \& Ettema, 2012), but not during maximal intensity of 1RM, where the TB (Clemons \& Aaron, 1997; Maszczyk et al., 2016) or AD (Król \& Gołaś, 2017) activity has been reported as a dominant muscle group for a successful lift.

Alternating details in exercise selection has been a very popular practice (Bishop, Chavda, \& Turner, 2018; Read, Bishop, Brazier, \& Turner, 2016). Our results indicate that the IMSP belongs to exercises targeting the TBlong with high activity such as the barbell pullover (Costa Campos \& da Silva, 2014), flat BP and narrow grip BP (Barnett et al., 1995). However, the TBlat is not significantly targeted in the IMSP as in the flat BP (51-68\% MVIC) where this muscle activity did not differ to TBlong (55-75\% MVIC) in females (Gołaś, Maszczyk, Pietraszewski, et al., 2018). The IMSP also targets very high $\mathrm{AD}$ activity such as the peck deck (Rocha, Gentil, Oliveira, \& Do Carmo, 2007), bench press (117.2\% MVIC; Santana et al., 2007), standing cable press (120.5\% MVIC; Santana et al., 2007), Smith machine BP (McCaw \& Friday, 1994; Schick et al., 2010), dumbbell press and the flat BP (Saeterbakken, van den Tillaar, \& Fimland, 2011). Therefore, we can conclude that all upper limb presses are exercises that significantly activate the AD.
Some of the EMG values in this study recorded during the exercise intensity $1 \mathrm{RM}$ and $80 \%$ of $1 \mathrm{RM}$ exceeded $100 \%$ MVIC, which is reasonable because the MVIC values were measured in an isometric (not SSC) condition. It is common to have dynamic EMG values above 100\% MVIC (Golas, Maszczyk, Stastny, et al., 2018; Hämäläinen \& Vanharanta, 1992; Netto \& Burnett, 2006; Oliver, 2014; Stastny et al., 2016), especially during high exercise intensity. The main limits of our study are the number of participants, the lack of speed control during concentric phase of the movement and absence of upper limb kinematic records (Stastny et al., 2017, Vigotski et al., 2017), which should be included in further studies aimed to analyze the IMSP.

\section{Conclusions}

The IMSP is an exercise which activated AD and TBlong more than PM and TBlat during maximal lifting effort. Therefore, the IMSP should be understood as the exercise which might substantially overload the $\mathrm{AD}$ and TBlong during maximal lifts in trained women. Coaches should use the IMSP at any intensity $60-100 \%$ of $1 \mathrm{RM}$ to exercise the TBlong without significant activity of TBlat to exhaust or overload TBlong more than TBlat during upper limb pressing.

\section{Acknowledgments}

This study was supported by the Charles University in Prague (grant no. UNCE/HUM/032).

\section{Conflict of interest}

There were no conflicts of interest.

\section{References}

Baechle, T. R., \& Earle, R. W. (2008). Essentials of strength training and conditioning (3rd ed.). Champaign, IL: Human Kinetics.

Barnett, C., Kippers, V., \& Turner, P. (1995). Effects of variations of the bench press exercise on the EMG activity of five shoulder muscles. Journal of Strength and Conditioning Research, 9, 222-227.

Bishop, C., Chavda, S., \& Turner, A. (2018). Exercise technique: The push press. Strength and Conditioning Journal, 40, 104-108.

Chandler, T. J., \& Brown, L. E. (2008). Conditioning for strength and human performance. New York, NY: Lippincott Williams \& Wilkins. 
Clemons, J. M., \& Aaron, C. (1997). Effect of grip width on the myoelectric activity of the prime movers in the bench press. Journal of Strength and Conditioning Research, 11, 82-87.

Cohen, J. (1988). Statistical power analysis for the behavioral sciences (2nd ed.). Hillsdale, NJ: Laurence Erlbaum Associates.

Costa Campos, Y., \& da Silva, S. F. (2014). Comparison of electromyographic activity during the bench press and barbell pullover exercises. Motriz: Revista de Educação Física, 20, 200-205.

Enoka, R. M., \& Duchateau, J. (2015). Inappropriate interpretation of surface EMG signals and muscle fiber characteristics impedes understanding of the control of neuromuscular function. Journal of Applied Physiology, 119, 1516-1518.

Gołaś, A., Maszczyk, A., Pietraszewski, P., Wilk, M., Stastny, P., Strońska, K., ... Zając, A. (2018). Muscular activity patterns of female and male athletes during the flat bench press. Biology of Sport, 35, 175-179.

Golas, A., Maszczyk, A., Stastny, P., Wilk, M., Ficek, K., Lockie, R. G., \& Zajac, A. (2018). A new approach to EMG analysis of closed-circuit movements such as the flat bench press. Sports, 6(2), 27.

Gołaś, A., Zwierzchowska, A., Maszczyk, A., Wilk, M., Stastny, P., \& Zając, A. (2017). Neuromuscular control during the bench press movement in an elite disabled and ablebodied athlete. Journal of Human Kinetics, 60, 209-215.

Hämäläinen, O., \& Vanharanta, H. (1992). Effect of Gz forces and head movements on cervical erector spinae muscle strain. Aviation, Space, and Environmental Medicine, 63, 709-716.

Hermens, H. J., Freriks, B., Merletti, R., Stegeman, D., Blok, J., Rau, G., ... Hägg, G. (1999). European recommendations for surface electromyography: Results of the SENIAM project. Enschede, Netherlands: Roessingh Research and Development.

Kholinne, E., Zulkarnain, R. F., Sun, Y. C., Lim, S., Chun, J.-M., \& Jeon, I.-H. (2018). The different role of each head of the triceps brachii muscle in elbow extension. Acta Orthopaedica et Traumatologica Turcica, 52, 201-205.

Król, H., \& Gołaś, A. (2017). Effect of barbell weight on the structure of the flat bench press. Journal of Strength and Conditioning Research, 31, 1321-1337.

Lantz, J., \& McCrain, M. (2005). Modifying chest press exercises for athletes with shoulder pathology. Strength and Conditioning Journal, 27, 69-72.

Larson-Hall, J. (2010). A guide to doing statistics in second language research using SPSS. Ibérica, 20, 167-204.

Lauver, J. D., Cayot, T. E., \& Scheuermann, B. W. (2016). Influence of bench angle on upper extremity muscular activation during bench press exercise. European Journal of Sport Science, 16, 309-316.

Lehman, G. J. (2005). The influence of grip width and forearm pronation/supination on upper-body myoelectric activity during the flat bench press. Journal of Strength and Conditioning Research, 19, 587-591.

Maszczyk, A., Golas, A., Czuba, M., Krol, H., Wilk, M., Kostrzewa, M., ... Goodwin, J. (2016). EMG analysis and modelling of Flat Bench Press using artificial neural networks. South African Journal for Research in Sport, Physical Education and Recreation, 38, 91-103.
McCaw, S. T., \& Friday, J. J. (1994). A comparison of muscle activity between a free weight and machine bench press. Journal of Strength and Conditioning Research, 8, 259-264.

Netto, K. J., \& Burnett, A. F. (2006). Reliability of normalisation methods for EMG analysis of neck muscles. Work, 26, 123-130.

Oliver, G. D. (2014). Relationship between gluteal muscle activation and upper extremity kinematics and kinetics in softball position players. Medical \& Biological Engineering \& Computing, 52, 265-270.

Read, P. J., Bishop, C., Brazier, J., \& Turner, A. N. (2016). Performance modeling: A system-based approach to exercise selection. Strength and Conditioning Journal, 38, 90-97.

Rocha, V. D. A. Jr., Gentil, P., Oliveira, E., \& Do Carmo, J. (2007). Comparison among the EMG activity of the pectoralis major, anterior deltoidis and triceps brachii during the bench press and peck deck exercises. Revista Brasileira de Medicina do Esporte, 13, 43e-46e.

Saeterbakken, A. H., van den Tillaar, R., \& Fimland, M. S. (2011). A comparison of muscle activity and 1-RM strength of three chest-press exercises with different stability requirements. Journal of Sports Sciences, 29, 533-538.

Santana, J. C., Vera-Garcia, F. J., \& McGill, S. M. (2007). A kinetic and electromyographic comparison of the standing cable press and bench press. Journal of Strength and Conditioning Research, 21, 1271-1277.

Schick, E. E., Coburn, J. W., Brown, L. E., Judelson, D. A., Khamoui, A. V., Tran, T. T., \& Uribe, B. P. (2010). A comparison of muscle activation between a Smith machine and free weight bench press. Journal of Strength and Conditioning Research, 24, 779-784.

Stastny, P., Bacakova, R., Chytilova, M., Tufano, J., Golas, A., \& Maszczyk, A. (2016). The differences in shoulder muscle activity between injured and uninjured rugby players during player-and bag-tackling. Trends in Sport Sciences, 4(23), 185-191.

Stastny, P., Gołaś, A., Blazek, D., Maszczyk, A., Wilk, M., Pietraszewski, P., ... Zając, A. (2017). A systematic review of surface electromyography analyses of the bench press movement task. PloS ONE, 12(2), e0171632.

Terzis, G., Karampatsos, G., \& Georgiadis, G. (2007). Neuromuscular control and performance in shot-put athletes. Journal of Sports Medicine and Physical Fitness, 47, 284-290.

Trebs, A. A., Brandenburg, J. P., \& Pitney, W. A. (2010). An electromyography analysis of 3 muscles surrounding the shoulder joint during the performance of a chest press exercise at several angles. Journal of Strength and Conditioning Research, 24, 1925-1930.

van den Tillaar, R., \& Sæterbakken, A. (2012). The sticking region in three chest-press exercises with increasing degrees of freedom. Journal of Strength and Conditioning Research, 26, 2962-2969.

van den Tillaar, R., Saeterbakken, A. H., \& Ettema, G. (2012). Is the occurrence of the sticking region the result of diminishing potentiation in bench press? Journal of Sports Sciences, 30, 591-599.

Vigotsky, A. D., Halperin, I., Lehman, G. J., Trajano, G. S., \& Vieira, T. M. (2017). Interpreting signal amplitudes in surface electromyography studies in sport and rehabilitation sciences. Frontiers in Physiology, 8, 985. 